\title{
Saffron for mild cognitive impairment and dementia: a systematic review and meta- analysis of randomised clinical trials
}

\author{
Zahra Ayati ${ }^{1,2}$, Guoyan Yang ${ }^{2}$, Mohammad Hossein Ayati ${ }^{3}$, Seyed Ahmad Emami ${ }^{1}$ and Dennis Chang ${ }^{2 *}$
}

\begin{abstract}
Background: Saffron (stigma of Crocus sativus L.) from Iridaceae family is a well-known traditional herbal medicine that has been used for hundreds of years to treat several diseases such as depressive mood, cancer and cardiovascular disorders. Recently, anti-dementia property of saffron has been indicated. However, the effects of saffron for the management of dementia remain controversial. The aim of the present study is to explore the effectiveness and safety of saffron in treating mild cognitive impairment and dementia.
\end{abstract}

Methods: An electronic database search of some major English and Chinese databases was conducted until 31st May 2019 to identify relevant randomised clinical trials (RCT). The primary outcome was cognitive function and the secondary outcomes included daily living function, global clinical assessment, quality of life (QoL), psychiatric assessment and safety. Rev-Man 5.3 software was applied to perform the meta-analyses.

Results: A total of four RCTs were included in this review. The analysis revealed that saffron significantly improves cognitive function measured by the Alzheimer's Disease Assessment Scale-cognitive subscale (ADAS-cog) and Clinical Dementia Rating Scale-Sums of Boxes (CDR-SB), compared to placebo groups. In addition, there was no significant difference between saffron and conventional medicine, as measured by cognitive scales such as ADAS-cog and CDR-SB. Saffron improved daily living function, but the changes were not statistically significant. No serious adverse events were reported in the included studies.

Conclusions: Saffron may have the potential to improve cognitive function and activities of daily living in patients with Alzheimer's disease and mild cognitive impairment (MCl). However, due to limited high-quality studies there is insufficient evidence to make any recommendations for clinical use. Further clinical trials on larger sample sizes are warranted to shed more light on its efficacy and safety.

Keywords: Dementia, Alzheimer's disease, MCl, Saffron, Iridaceae, Systematic review

\section{Background}

Mild cognitive impairment (MCI) is defined by mild quantifiable decline in cognitive function greater than expected for an individual's age and education level, but essentially preserved functional abilities [1-3]. It is

\footnotetext{
* Correspondence: D.Chang@westernsydney.edu.au

${ }^{2} \mathrm{NICM}$ Heath Research Institute, Western Sydney University, Westmead, NSW 2145, Australia

Full list of author information is available at the end of the article
}

regarded as a risk state for dementia. The estimated prevalence of MCI in population-based studies ranges from 10 to $20 \%$ in people older than 65 years of age. Currently, there is no proven effective medication in treatment of MCI [4].

Dementia is a major cognitive disorder which is characterized by any significant cognitive decline from a previously higher level of functioning and compromises social and/or occupational functions [5]. Alzheimer's

(c) The Author(s). 2020 Open Access This article is licensed under a Creative Commons Attribution 4.0 International License, which permits use, sharing, adaptation, distribution and reproduction in any medium or format, as long as you give appropriate credit to the original author(s) and the source, provide a link to the Creative Commons licence, and indicate if changes were made. The images or other third party material in this article are included in the article's Creative Commons licence, unless indicated otherwise in a credit line to the material. If material is not included in the article's Creative Commons licence and your intended use is not permitted by statutory regulation or exceeds the permitted use, you will need to obtain permission directly from the copyright holder. To view a copy of this licence, visit http://creativecommons.org/licenses/by/4.0/ The Creative Commons Public Domain Dedication waiver (http://creativecommons.org/publicdomain/zero/1.0/) applies to the data made available in this article, unless otherwise stated in a credit line to the data. 
disease $(\mathrm{AD})$ is the most common neurodegenerative disorder and the most prevalent cause of dementia worldwide with an estimated prevalence of $10-30 \%$ in those aged $>65$ years [6-8]. It is characterized by cognitive decline with loss of memory [9]. No pharmaceutical medicines are currently available to cure dementia, although some medications such as memantine ( $\mathrm{N}$-methyl-D-aspartate (NMDA) channel blocker), and galantamine, donepezil and rivastigmine (cholinesterase inhibitors) are used clinically to manage symptoms of the disease. However, these medications have some side effects such as headache, confusion, nausea and vomiting and leg cramp which are reported to be intolerable in some patients $[10$, 11].

Crocus sativus (saffron) which belongs to the Iridaceae family has a long history of use as a spice, colouring agent and herbal medicine since ancient times. It has been suggested that saffron is effective for several diseases such as depression, respiratory and cardiovascular disorders [12]. Saffron has also been used in traditional Persian and Chinese medicines to restore and enhance memory [13-15]. Recent clinical trials [16-19] and reviews [20-32] revealed the positive effects of saffron on cognitive deterioration and improving functional and behavioural disturbances in patients with dementia and MCI. However, the efficacy of saffron on dementia and MCI is still unclear. Although saffron has been used as a food additive for many centuries implying its safety in human consumption, the toxicity and safety of saffron requires careful evaluation when used as a medicinal herb. Several clinical trials have directly evaluated the safety of saffron. Saffron with doses less than $1.5 \mathrm{~g} /$ daily is considered relatively safe in healthy humans, however, toxic effects are reported with doses $5 \mathrm{~g}$ /daily and above with a lethal dose of about $20 \mathrm{~g} /$ daily [31]. The safety of saffron in MCI and dementia needs to be further investigated given the reduced renal and hepatic functions in these elderly cohorts.

To the best of our knowledge, no systematic reviews are yet conducted to assess the efficacy and safety of saffron for MCI and dementia. Accordingly, we aimed to review the effectiveness and safety of saffron for the treatment of MCI and dementia systematically and perform a meta-analysis to assess the magnitude of these effects, when possible.

\section{Methods}

This systematic review is registered in PROSPERO, International prospective register of systematic reviews (Registration number: CRD42019127560). We followed the Preferred Reporting Items for Systematic Reviews and Meta-Analysis Statement (PRISMA) guidelines [33] to ensure comprehensive and transparent reporting of methods and results.

\section{Literature search and study selection}

Two authors (ZA and GYY) independently conducted literature search from Web of Science, Pub-Med, Scopus, EMBASE and Cochrane Library, and two authors (GYY and MHA) independently conducted literature search from four major Chinese databases including China Network Knowledge Infrastructure (CNKI), Chinese Scientific Journals Database (VIP), Sino-Med Database and Wan Fang Database, from their inception until 31st May, 2019.

The English searching terms included "Crocus sativus", "Saffron", "Croci Stigma", "iridaceae", "Zafran" and "Alzheimer", "mild cognitive impairment", "senile dementia", and "random". The Chinese searching terms included mild cognitive impairment ("qing_du_ren_zhi zhang_ai" and "qing_du_ren_zhi_gong_neng_zhang_ai"), Alzheimer's disease ("a_er_ci_hai_mo", "a_er_ci_hai mo_bing", "a_er_zi_hai_mo", "a_er_zi_hai_mo_bing", "lao_nian_chi_dai", "a_er_cai_mu_shi", "lao_nian_xing chi_dai" and "lao_nian_qi_chi_dai"), saffron ("hong $h u a$ "), and randomized ("sui_ji").

Two authors (ZA and GYY) independently screened the potential titles and abstracts according to the inclusion and exclusion criteria. The full texts of potentially eligible articles were retrieved and independently assessed by two authors (ZA and GYY) for eligibility. Any discrepancies were identified and resolved through discussion with a third author (DC). To assure inclusion of all relevant papers, reference lists of primary extracted studies and review papers were independently hand searched by two reviewers.

\section{Inclusion and exclusion criteria Study design}

Randomised controlled trials (either parallel or cross-over designs), with at least one group involving saffron for the treatment of MCI or dementia (including Alzheimer's disease, vascular disease, Lewy body dementia, mixed dementia, Parkinson's disease related dementia, and frontotemporal dementia) regardless of severity were included. For cross-over trials, only the outcomes of the first period were included. Other types of human studies such as quasi-randomized trials were excluded.

\section{Participants}

Participants diagnosed with any one of the following criteria as mild cognitive impairment were included: (a) The Diagnostic and Statistical Manual of Mental Disorder (DSM) III, III-R or IV; (b) The International Classification of Disease (ICD) version 9 or 10; (c) Petersen criteria; (d) European Consortium on Alzheimer's disease.

Participants diagnosed with any one of the following criteria as dementia were included, regardless of severity and disease course: (a) The Diagnostic and Statistical 
Manual of Mental Disorder (DSM) III, III-R or IV; (b) The International Classification of Disease (ICD) (9th or 10th edition); (c) The National Institute of Neurological and Communicative Disorder and Stroke-Alzheimer's Disease and Related Disorder Association (NINCDS/ ADRDA).

Participants diagnosed with any of the following criteria as Vascular dementia were included: (a) DSM-III, III-R or IV criteria for the diagnosis of vascular dementia (b) The National Institute of Neurological Disorders and Stroke (NINDS) and the Association Internationale pour la Recherche et l'Enseignement en Neurosciences (AIRE $\mathrm{N})$ criteria for the diagnosis of vascular dementia.

\section{Interventions}

Any forms of saffron (powder, extract, or oil) were included. Studies that examined saffron in combination with other ingredients and studies which evaluated the effect of an active component of saffron were excluded.

\section{Control}

Studies that compared saffron with placebo, no treatment and conventional treatments were included. Cointerventions were also allowed, if applied in all arms.

\section{Outcome measures}

The primary outcome was cognitive function. The secondary outcomes included activities of daily living $(\mathrm{ADL})$, quality of life (QoL), global clinical assessment, daily living function, psychiatric assessment and safety.

\section{Data extraction}

A standardised, pre-piloted form was used to extract data from the included studies for assessment of study quality and evidence synthesis. Data to be extracted were as follows: study population and baseline characteristics, interventions and control condition, dosage and duration of intervention, outcome measures, and main results.

If relevant information was not available in the paper, corresponding authors of the papers were contacted via email three times at reasonable intervals.

\section{Quality assessment}

The risk of bias was evaluated independently by two authors (ZA and GYY) using the Cochrane collaboration recommended tool [34]. We assessed six biases accordingly, including: selection bias (random sequence generation and allocation concealment), performance bias and detection bias (blinding), attrition bias (incomplete outcome data), reporting bias (selective outcome reporting) and other bias. We categorised each item in to "low", "unclear" or "high risk" of bias. If a trial met all criteria, a low risk of bias was given; if a trial met none of the criteria, a high risk of bias was given; and if a trial provided insufficient information to judge, unclear risk of bias was given. Any disagreement about the judgment of the risk of bias was discussed and resolved by involving a third author (DC).

\section{Statistical analysis}

Meta-analyses were performed using RevMan 5.3 software. Data were summarised by odds ratios (OR) with 95\% confidence intervals $(\mathrm{Cl})$, and data for continuous outcomes were performed using mean difference (MD) with 95\% CI. Heterogeneity among trials was detected using I-squared $\left(I^{2}\right)$ index. $I^{2}$ values greater than $50 \%$ were regarded as high heterogeneity. Random-effects model was used to conduct the meta-analysis unless the $I^{2}$ statistic was less than $25 \%$. We did not perform funnel plots to detect publication bias because there were less than 10 trials under each outcome.

\section{Results}

The major English and Chinese databases were searched from their inception till 31st May 2019. The search returned 191 results which reduced to 145 after duplicates were removed. By screening the titles and abstracts for eligibility, a further 135 articles were excluded. After reviewing the full texts of the remaining 10 papers, 6 were further excluded for the following reasons: saffron was used in combination with other herbs or vitamins $(n=5)$ and saffron was evaluated on non-dementia or MCI patients $(n=1)$. Full details of search results are summarised in Fig. 1.

\section{Characteristics of the included studies}

A total of 4 trials [16-19] with 203 patients were eligible for inclusion. All the included trials were 2-armed studies and published in English language. Three of the trials had been conducted in Iran and one in Greece. Among the 4 trials, 3 trials [16-18] focused on $\mathrm{AD}$ and the other trial [19] focused on MCI. No trial on other types of dementia was found.

The duration of treatments varied from 4 to 12 months. All the trials on AD used standard saffron extract $30 \mathrm{mg}$ per day and the trial on MCI used saffron powder $125 \mathrm{mg}$ daily. The comparison of saffron with placebo was performed in one trial [17], with conventional medicine in 2 trials $[16,18]$, and with no positive control or placebo in one trial [19]. Table 1 presents the detailed characteristics of the included trials.

\section{Risk of bias assessment}

For random sequence generation, three trials [16-18] used computer-based randomisation and the remaining one just simply mentioned "randomized normal distribution" and did not report the specific method of random sequence generation. 


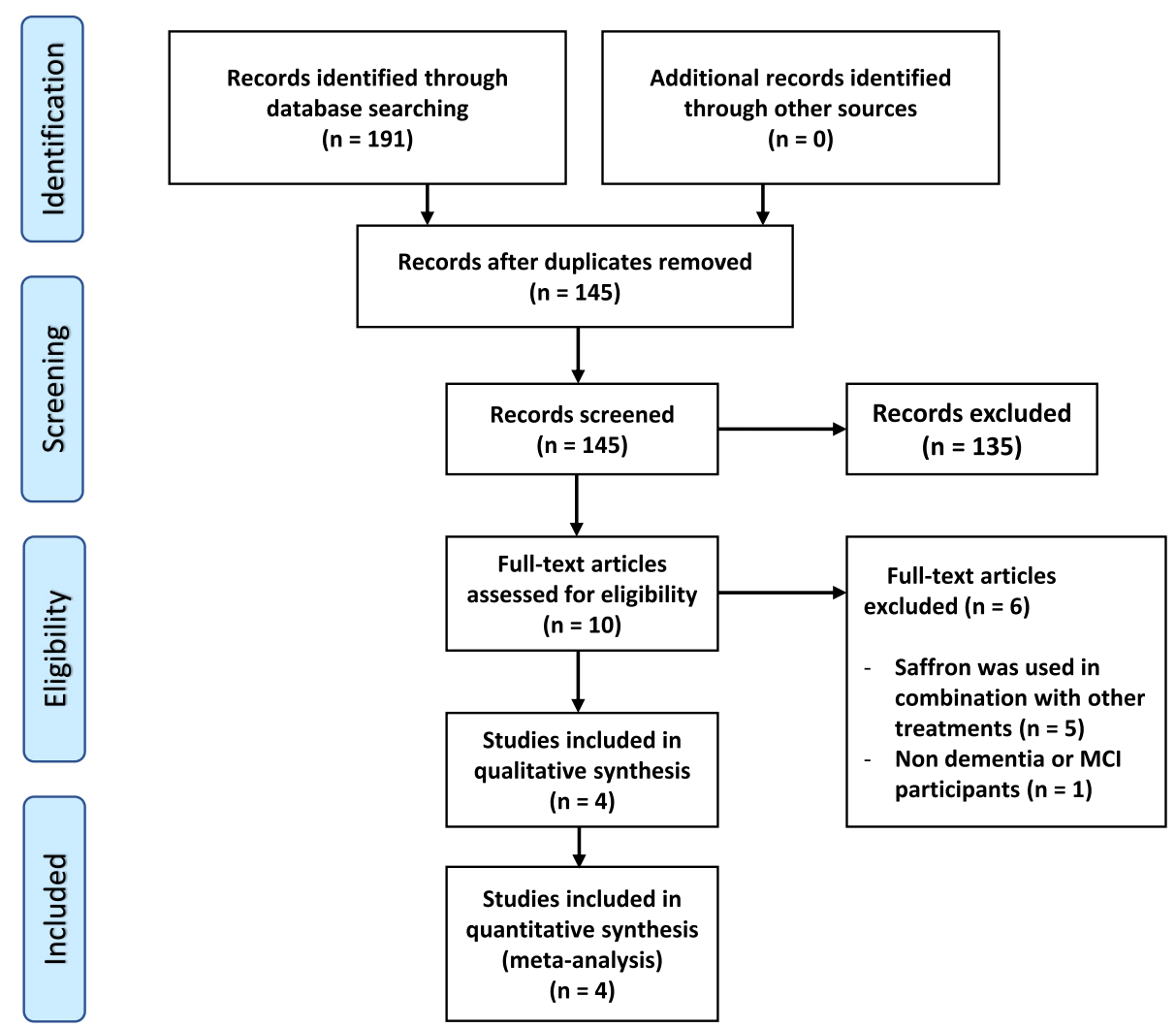

Fig. 1 PRISMA flow diagram

Regarding the allocation concealment, 3 of the trials reported the allocation concealment method in detail [16-18] and the remaining one did not report any information about it. Three trials were double-blind trials (all on AD) and one was single blind (on MCI). Three trials (all on $\mathrm{AD}$ ) described the number and reasons of withdrawal, while the remaining one did not report this information.

The general methodological quality of the trials of saffron for $\mathrm{AD}$ was moderate to high and for the trial of saffron on MCI was poor (Figs. 2 and 3).

\section{Findings from systematic review and meta-analysis The effect of saffron on cognitive function}

Three studies on AD reported this outcome [16-18]. Compared with placebo, one study by Akhondzadeh et al. [17] found that saffron improved ADAS-cog and CDR-SB scores significantly over 16 weeks. Compared with conventional medicine (donepezil), another study by Akhondzadeh et al. [16] demonstrated that saffron improved Alzheimer's Disease Assessment Scale-cognitive subscale (ADAS-cog) and Clinical dementia rating scale-sums of boxes (CDR-SB) scores at 22 weeks, and the changes were statistically comparable between groups. Compared with conventional medicine (memantine), one study by Farokhnia et al. [18] revealed that saffron treatment caused an improvement in Mini-Mental State Examination (MMSE) and Severe Cognitive Impairment Rating Scale (SCIRS) scores at 48 weeks, and it was statistically comparable between groups (Table 2).

One study on MCI also reported this outcome. Tsolaki et al. [19] found that saffron compared with "no treatment" was superior in improving cognitive function; the change in MMSE score in the saffron group was higher than that of the "no treatment" group. An improvement in Montreal Cognitive Assessment (MoCA) score was also observed in the saffron group compare to "no treatment" but the changes in MMSE and MoCA failed to reach statistical significance (Table 2). Additionally, the magnetic resonance imaging (MRI) results showed a small difference in the volume of left inferior temporal gyrus in favour of the saffron group [19].

\section{The effect of saffron on daily living function}

Daily living function in response to saffron treatment was evaluated in two studies $[18,19]$. In the study on dementia by Farokhnia et al. [18], saffron treatment over 12 months improved Functional Assessment Staging (FAST) scale by -0.03 when compared to conventional medicine (memantine) and no significant difference was found (Table 2). 


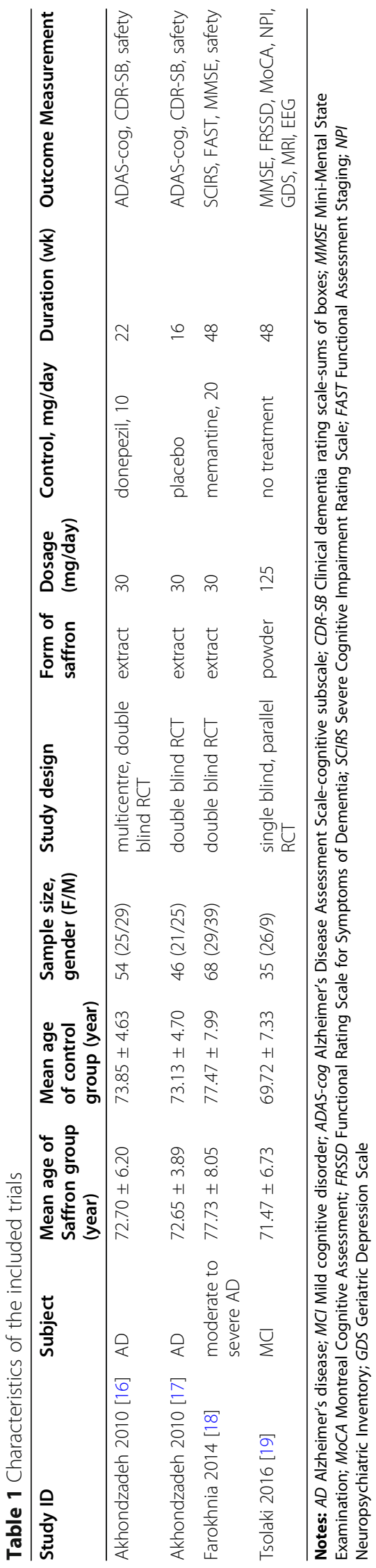




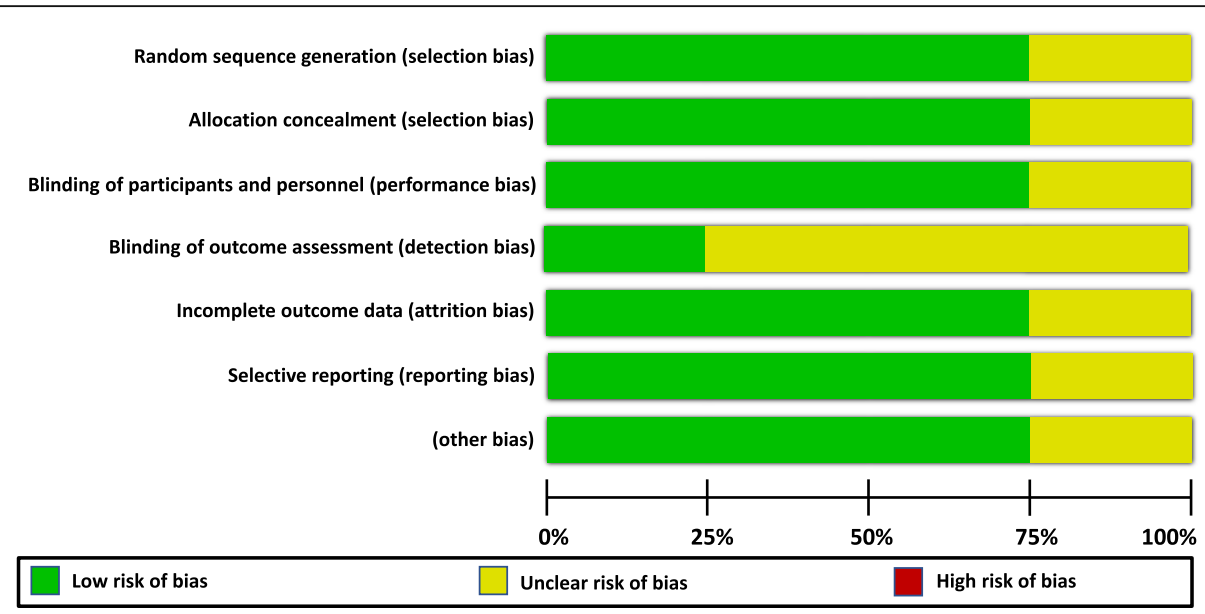

Fig. 2 Risk of bias graph of the randomised controlled trials on saffron for mild cognitive impairment $(M C l)$ and dementia
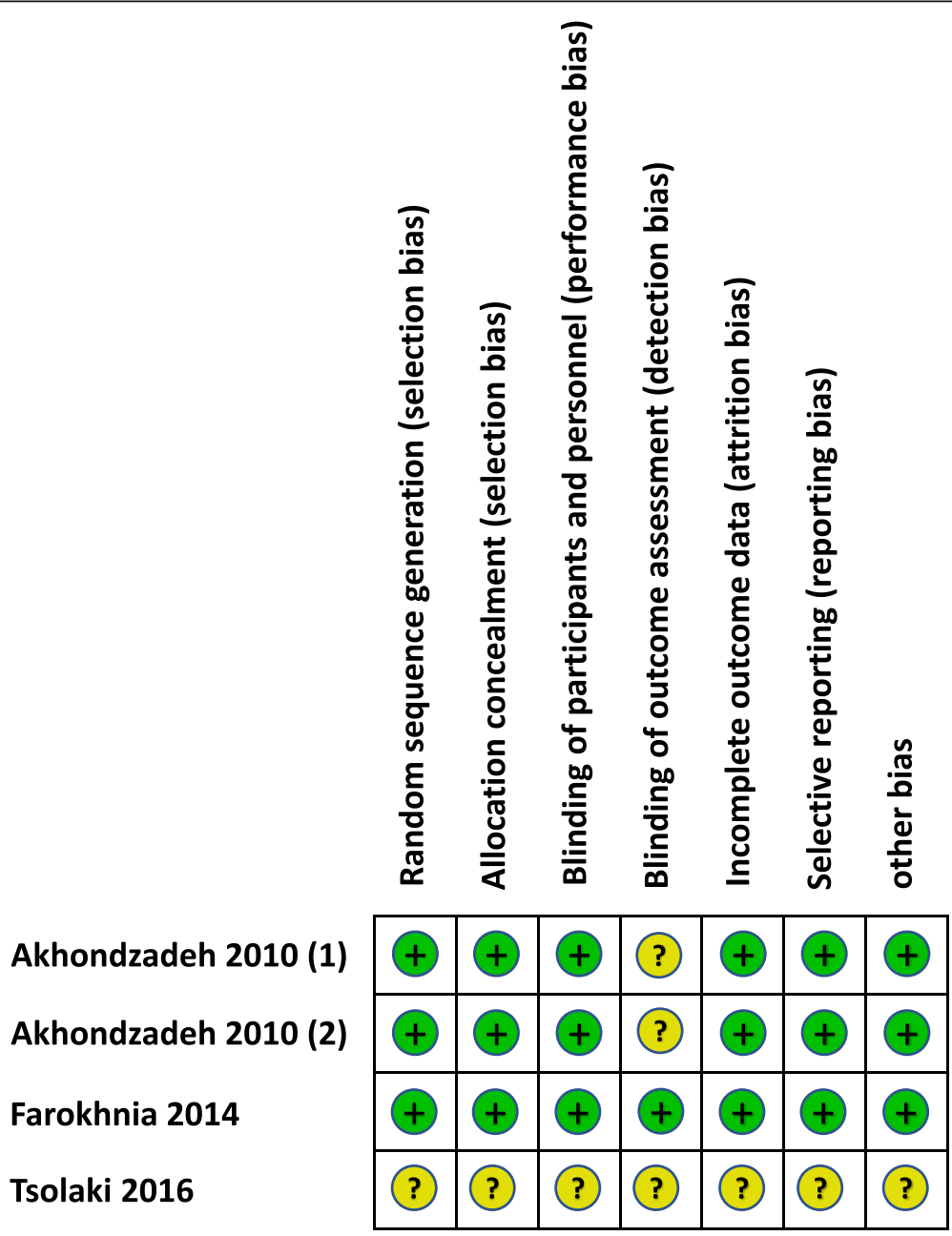

Fig. 3 Risk of bias summary of the randomised controlled trials on saffron for mild cognitive impairment (MCI) and dementia 
Table 2 Effect estimate for saffron on cognitive function, daily living function and psychological parameters

\begin{tabular}{|c|c|c|c|c|}
\hline Outcome & Number of studies & Number of participants & $\begin{array}{l}\text { Effect estimate by mean difference } \\
\text { (IV, Random, } 95 \% \mathrm{Cl}), p \text { value }\end{array}$ & Study ID \\
\hline \multicolumn{5}{|c|}{ Cognitive function } \\
\hline ADAS-cog $^{1}$ & 1 & 47 & $-0.19[-2.28,1.90], 0.86$ & Akhondzadeh 2010 [17] \\
\hline ADAS-cog ${ }^{2}$ & 1 & 42 & $-7.77[-8.69,-6.85],{ }^{*} p<0.00001$ & Akhondzadeh 2010 [16] \\
\hline $\mathrm{CDR}_{-} \mathrm{SB}^{1}$ & 1 & 47 & $0.06[-0.49,0.61], 0.83$ & Akhondzadeh 2010 [17] \\
\hline $\mathrm{CDR}_{-} \mathrm{SB}^{2}$ & 1 & 42 & $-1.30[-1.52,-1.08],{ }^{*} p<0.00001$ & Akhondzadeh 2010 [16] \\
\hline MMSE $^{a}$ & 1 & 60 & $-0.38[-1.12,0.36], 0.32$ & Farokhnia 2014 [18] \\
\hline MMSE $^{b}$ & 1 & 35 & $1.07[-0.36,2.50], 0.14$ & Tsolaki 2016 [19] \\
\hline SCIRS & 1 & 60 & $0.27[-0.36,0.90], 0.40$ & Farokhnia 2014 [18] \\
\hline MoCA & 1 & 35 & $1.48[-1.15,4.11], 0.27$ & Tsolaki 2016 [19] \\
\hline \multicolumn{5}{|c|}{ Daily living function } \\
\hline FRSSD & 1 & 35 & $-1.13[-3.44,1.18], 0.34$ & Tsolaki 2016 [19] \\
\hline FAST & 1 & 60 & $-0.03[-0.43,0.37], 0.88$ & Farokhnia 2014 [18] \\
\hline \multicolumn{5}{|c|}{ Psychiatric assessment } \\
\hline NPI & 1 & 35 & $-3.37[-8.41,1.67], 0.19$ & Tsolaki 2016 [19] \\
\hline GDS & 1 & 35 & $0.30[-1.99,2.59], 0.80$ & Tsolaki 2016 [19] \\
\hline
\end{tabular}

Note: *, statistically significant; 1 , saffron compared with conventional medicine; 2, saffron compared with placebo; a, comparison of the changes from baseline to the end point between saffron and conventional group; $b$, comparison of the post scores between saffron and control group

Also, a 1-year saffron treatment reduced the score of Functional Rating Scale for Symptoms of Dementia (FRSSD) in MCI patients compare to no treatment (Tsolaki et al., [19]). However, this change was not statistically significant (Table 2).

\section{Psychological assessment}

In study by Tsolaki et al. [19], the Neuropsychiatric Inventory (NPI) score was improved after a one-year administration of saffron compare to the control group. In the same study, Geriatric Depression Scale (GDS) score was slightly higher at the end of trial in the saffron group when compared to that of control, but the changes in NPI and GDS are not statistically significant (Table 2).

\section{Adverse events}

Out of the 4 included trials, all 3 studies on AD reported safety information and the number of dropouts. One of the trials [17] which compared saffron with placebo reported one death in the control group due to myocardial infarction and mild adverse events such as dizziness, dry mouth, fatigue and nausea were reported in both saffron and control groups; no between group difference was found. In the other two trials $[16,18]$ that compared saffron with conventional medicine, mild adverse effects such as nausea, dry mouth and fatigue were reported and the differences between two groups were not statistically significant (Table 3). In one of these studies [16], one death was reported in the donepezil group due to myocardial infarction.

Table 3 Meta-analysis results for adverse events of saffron

\begin{tabular}{llll}
\hline Adverse events & Number of studies & Number of participants & Effect estimate (Odds Ratio (M-H, Random, 95\% Cl), $\boldsymbol{p}$ value \\
\hline Nausea & 3 & 156 & $0.90[0.29,2.80], 0.85$ \\
Dry mouth & 3 & 156 & $1.53[0.58,4.07], 0.39$ \\
Fatigue & 3 & 156 & $0.52[0.17,1.53], 0.23$ \\
Dizziness & 3 & 156 & $0.51[0.20,1.31], 0.16$ \\
Confusion & 1 & 60 & $1.00[0.06,16.76], 1$ \\
Agitation & 1 & 60 & $0.19[0.01,4.06], 0.29$ \\
Sedation & 1 & 60 & $0.31[0.03,3.17], 0.32$ \\
Vomiting & 2 & 114 & $0.47[0.04,6.05], 0.56$ \\
Hypomania & 2 & 96 & $3.990 .42,37.49], 0.23$ \\
\hline
\end{tabular}


Rates of patients remaining in the trials until the end were $90.47 \%$ for the saffron groups, $86.88 \%$ for conventional treatment groups and $86.95 \%$ for other control groups (placebo and no-treatment).

\section{Discussion}

Saffron appears to be beneficial to cognitive performance in patients with MCI and AD. However, due to the small number of included clinical trials with diverse outcome measurements, we could not draw a definitive conclusion.

To the best of our knowledge, this is the first systematic review that examines the effects of saffron on cognitive performance in patients with MCI or dementia. No papers were identified to evaluate the effects of saffron on other types of dementia such as vascular dementia.

The general methodological quality of the three included trials on $\mathrm{AD}$ was high; Information on registration and the specific method of random sequence generation, allocation concealment and blinding were reported. However, the methodological quality of the only trial on MCI was poor due to the lack of information on the specific method of random sequence generation, allocation concealment, blinding, missing data and trial registration or protocol publication.

All included trials demonstrated that saffron had potential benefits in improving cognitive function for the treatment of $\mathrm{AD}$ and $\mathrm{MCI}$. When measured by ADAS$\operatorname{cog}$ and $\mathrm{CDR}-\mathrm{SB}$, treatments with saffron caused a clinically significant improvement compared with placebo. The magnitude of the changes in ADAS-cog appear to be high as it is in general agreement that a four-unit change on the ADAS-cog is required for a clinically significant/meaningful improvement [35]. In addition, compared with conventional medicine, the between group difference was not significant as measured by cognitive scales including ADAS-cog, CDR-SB and SCIRS.

The effect of saffron for daily living functioning is still in doubt. Although saffron treatment demonstrated a trend towards improvement in FAST and FRSSD, these changes were not significant.

Rates of retention and adherence of participants in $\mathrm{AD}$ trials were high in saffron groups and was slightly higher than that of the control groups. The safety profile of saffron appeared to be good. No serious adverse event (SAE) was reported in the saffron treatment groups. Mild adverse events such as nausea, fatigue, dry mouth and dizziness were observed in both intervention and control groups. However, the results show that the prevalence of the side effects observed between the intervention and control groups was not statistically significant.

The doses of saffron extracts used in the three included AD trials were the same ( $30 \mathrm{mg}$ hydro alcoholic extract daily). The saffron extracts used in the $A D$ clinical trials were standardised by safranal and crocin which are two of the most bioactive constituents of saffron. Each capsule contained $1.65-1.75 \mathrm{mg}$ crocin and $0.13-0.15 \mathrm{mg}$ safranal in all AD clinical trials. In the MCI trial, saffron powder (125 mg/day) was used and no information on the standardisation was provided. According to the previous studies, toxic dose of saffron powder is greater than $5 \mathrm{~g} /$ day $[36,37]$ and therefore the dose used in this study appears to be safe. However, the quality of saffron in the MCI trial is not clear.

The exact mechanisms underlying the effects of saffron on dementia remain unclear. However, transe-crocin-4, a main carotenoid constituent of saffron has shown to be able to inhibit A-beta fibrillogenesis formation [38]. Abeta fibrillogenesis is formed by oxidation of amyloid beta-peptide fibrils and plays a significant role in the pathophysiology of AD. Additionally saffron extract has been shown to possess a moderate inhibitory activity $(30 \%)$ on acetyl-cholinesterase (AChE) and inhibits acetylcholine breakdown which is one of the main therapeutic targets for $\mathrm{AD}$ [39]. In an in vivo study, saffron also showed to increase antioxidant enzymes and decrease plasma levels of corticosterone suggesting that saffron is effective in improving the oxidative stress damage to the hippocampus followed by chronic stress [40].

In summary, only four trials were eligible to be included in this review using comprehensive search strategy both in English and Chinese databases with broad inclusion criteria. Although the methodological strength of the most included trials was reasonably strong, the number of participants in the included trials is low. A diverse outcome measures were used in these studies with different comparisons and it was not possible to conduct meta-analysis. Nevertheless, saffron has been shown to significantly improve ADAS-cog and CDR-SB scores in AD patients compared to placebo and was comparable to conventional medicine in improving cognitive function. However, given the small number of included trials, the clinical significance of these findings is in doubt.

\section{Conclusion}

The results of this review suggest that saffron may be beneficial to improve cognitive function in patients with $\mathrm{MCI}$ and AD. No evidence was found to support the effects of saffron on other types of dementia. More highquality randomised placebo-control trials are needed to further confirm the efficacy and safety of saffron for $\mathrm{MCI}$ and dementia.

\section{Abbreviations}

AD: Alzheimer's disease; AChE: Acetyl-cholinesterase; ADAS-cog: Alzheimer's disease assessment scale-cognitive subscale; ADL: Activities of daily living; AIREN: Association internationale pour la recherche et l'enseignement en neurosciences; CDR-SB: Clinical dementia rating scale-sums of boxes; Cl: Confidence intervals; CNKI: China network knowledge infrastructure; DSM: Diagnostic and statistical manual of mental disorder; FAST: Functional 
assessment staging; FRSSD: Functional rating scale for symptoms of dementia; GDS: Geriatric depression scale; ICD: International classification of disease; MCl: Mild cognitive impairment; MRI: Magnetic resonance imaging; MD: Mean difference; NINCDS/ADRDA: National institute of neurological and communicative disorder and stroke-alzheimer's disease and related disorder association; NINDS: National institute of neurological disorders and stroke; NPI: Neuropsychiatric inventory; PRISMA: Preferred reporting items for systematic reviews and meta-analysis statement; PROSPERO: International prospective register of systematic reviews; QoL: Quality of life; SAE: Severe adverse event

\section{Acknowledgments}

This work is supported by NICM Health Research Institute and Mashhad University of Medical Sciences. We gratefully acknowledge the help provided by Dr. Nazli Namazi and Dr. Joseph Firth for their valuable suggestions.

\section{Authors' contributions}

DC, GY and ZA provided the conception and design. ZA and GYY conducted literature search from the English databases. GYY and MHA conducted literature search from the Chinese databases. ZA and GYY carried out data collection and meta-analysis. ZA took the lead in writing the manuscript. DC and SAE supervised the project and provided critical review of the manuscript. All authors approved the final submission.

\section{Funding}

Not to be declared.

\section{Availability of data and materials}

The datasets used and analysed during the current study are available from the corresponding author on reasonable request.

\section{Ethics approval and consent to participate}

Not applicable.

\section{Consent for publication}

Not applicable.

\section{Competing interests}

As a medical research institute, NICM Health Research Institute receives research grants and donations from foundations, universities, government agencies, individuals and industry. Sponsors and donors also provide untied funding for work to advance the vision and mission of the Institute. The authors declare no competing financial interests.

\section{Author details}

'Department of Traditional Pharmacy, School of Pharmacy, Mashhad University of Medical Sciences, Mashhad, Iran. ${ }^{2}$ NICM Heath Research Institute, Western Sydney University, Westmead, NSW 2145, Australia. ${ }^{3}$ School of Traditional Medicine, Tehran University of Medical Sciences, Tehran, Iran.

Received: 12 December 2019 Accepted: 1 October 2020

Published online: 09 November 2020

\section{References}

1. Meyer JS, Xu G, Thornby J, Chowdhury MH, Quach M. Is mild cognitive impairment prodromal for vascular dementia like Alzheimer's disease? Stroke. 2002;33(8):1981-5.

2. Langa KM, Levine DA. The diagnosis and management of mild cognitive impairment: a clinical review. JAMA. 2014;312(23):2551-61.

3. Gauthier S, Reisberg B, Zaudig M, Petersen RC, Ritchie K, Broich K, Belleville S, Brodaty H, et al. Mild cognitive impairment. Lancet. 2006;367(9518):1262-70.

4. Petersen RC. Mild cognitive impairment. N Engl J Med. 2011;364(23):2227-34.

5. Hugo J, Ganguli M. Dementia and cognitive impairment: epidemiology, diagnosis, and treatment. Clin Geriatr Med. 2014;30(3):421-42.

6. Masters CL, Bateman R, Blennow K, Rowe CC, Sperling RA, Cummings JL Alzheimer's disease. Nat Rev Dis Primers. 2015;1:15056.

7. Scarpini E, Schelterns P, Feldman H. Treatment of Alzheimer's disease; current status and new perspectives. Lancet Neurol. 2003;2(9):539-47.

8. Association As. 2018 Alzheimer's disease facts and figures. Alzheimers Dement. 2018;14(3):367-429.
9. Angelucci F, Spalletta G, di lulio F, Ciaramella A, Salani F, Colantoni L, Varsi AE, Giann W, Sancesario G, Caltagirone C, Bossu P. Alzheimer's disease (AD) and mild cognitive impairment (MCl) patients are characterized by increased BDNF serum levels. Curr Alzheimer Res. 2010;7(1):15-20.

10. Alzheimer's Association. FDA-Approved Treatment for Alzheimer's. https:// alz.org/media/Documents/fda-approved-treatments-alzheimers-ts.pdf. Accessed March 2019

11. Livingston G, Sommerlad A, Orgeta V, Costafreda SG, Huntley J, Ames D, Ballard C, Banerjee S, Burns A, Cohen-Mansfield J, Cooper C, Fox N, Giltin $L N$, Howard R, et al. Dementia prevention, intervention, and care. Lancet. 2017;390(10113):2673-734.

12. Javadi B, Sahebkar A, Emami SA. A survey on saffron in major Islamic traditional medicine books. Iran J Basic Med Sci. 2013;16(1):1.

13. Hajiaghaee $\mathrm{R}$, Akhondzadeh $\mathrm{S}$. Herbal medicine in the treatment of Alzheimer's disease. J Med Plants. 2012:1(41):1-7.

14. Howes M-JR, Fang R, Houghton PJ. Effect of Chinese herbal medicine on Alzheimer's disease. Int Rev Neurobiol. 2017:135:29-56.

15. Rahimi R, Irannejad S, Noroozian M. Avicenna's pharmacological approach to memory enhancement. Neurol Sci. 2017;38(7):1147-57.

16. Akhondzadeh S, Sabet MS, Harirchian MH, Togha M, Cheraghmakani H, Razeghi S, Hejazi SS, Yousefi MH, Alimardani R, Jamshidi A, Rezazadeh SA, Yousefi A, Zare F, Moradi A, Vossoughi A. A 22-week, multicenter, randomized, double-blind controlled trial of Crocus sativus in the treatment of mild-tomoderate Alzheimer's disease. Psychopharmacology. 2010;207(4):637-43.

17. Akhondzadeh S, Sabet MS, Harirchian MH, Togha M, Cheraghmakani H, Razeghi S, Hejazi SS, Yousefi MH, Alimardani R, Jamshidi A, Zare F, Moradi A. Saffron in the treatment of patients with mild to moderate Alzheimer's disease: a 16-week, randomized and placebo-controlled trial. J Clin Pharm Ther. 2010;35(5):581-8.

18. Farokhnia M, Shafiee Sabet M, Iranpour N, Gougol A, Yekehtaz H, Alimardan R, Farsad F, Kamalipour M, Akhondzadeh S. Comparing the efficacy and safety of Crocus sativus $L$. with memantine in patients with moderate to severe Alzheimer's disease: a double-blind randomized clinical trial. Hum Psychopharmacol. 2014:29(4):351-9.

19. Tsolaki M, Karathanasi E, Lazarou I, Dovas K, Verykouki E, Karacostas A, Georgiadis K, Tsolaki A, Adam K, Kompatsiaris I, Sinakos Z. Efficacy and safety of crocus sativus $L$. in patients with mild cognitive impairment: one year single-blind randomized, with parallel groups, clinical trial. J Alzheimers Dis. 2016;54(1):129-33.

20. Adalier N, Parker H. Vitamin E, turmeric and saffron in treatment of alzheimer's disease. Antioxidants. 2016;5(4):40. https://doi.org/10.3390/ antiox5040040.

21. Akram M, Nawaz A. Effects of medicinal plants on alzheimer's disease and memory deficits. Neural Regen Res. 2017;12(4):660-70.

22. Berger $F$, Hensel A, Nieber K. Crocus sativus L. is not just a spice: Neuroprotective effect of saffron extract. Z Phytother. 2012;33(6):263-71.

23. Chang D, Liu J, Bilinski K, Xu L, Steiner GZ, Seto SW, Bensoussan A. Herbal medicine for the treatment of vascular dementia: An overview of scientific evidence. Evidence-based Complementary and Alternative Medicine. 2016: article number 7293626, 2016.

24. Christodoulou E, Kadoglou NP, Kostomitsopoulos N, Valsami G. Saffron: a natural product with potential pharmaceutical applications. J Pharm Pharmacol. 2015:67(12):1634-49.

25. Cicero AFG, Fogacci F, Banach M. Botanicals and phytochemicals active on cognitive decline: the clinical evidence. Pharmacol Res. 2018;130:204-12.

26. Finley JW, Gao S. A perspective on Crocus sativus L. (saffron) constituent crocin: a potent water-soluble antioxidant and potential therapy for Alzheimer's disease. J Agric Food Chem. 2017;65(5):1005-20.

27. Hajiaghaee $\mathrm{R}$, Akhondzadeh $\mathrm{S}$. Herbal medicine in the treatment of Alzheimer's disease. J Med Plants. 2012;11(41):1-7.

28. Leone S, Recinella L, Chiavaroli A, Orlando G, Ferrante C, Leporini L, et al. Phytotherapic use of the Crocus sativus L. (saffron) and its potential applications: a brief overview. Phytother Res. 2018;32(12):2364-75.

29. Milajerdi A, Bitarafan V, Mahmoudi M. A review on the effects of saffron extract and its constituents on factors related to neurologic, cardiovascular and gastrointestinal diseases. J Med Plants. 2015;14(55):9-28.

30. Mirmosayyeb O, Tanhaei A, Sohrabi HR, Martins RN, Tanhaei M, Najafi MA, et al. Possible role of common spices as a preventive and therapeutic agent for Alzheimer's disease. Int J Prev Med. 2017;8(1):5.

31. Moshiri M, Vahabzadeh $\mathrm{M}$, Hosseinzadeh $\mathrm{H}$. Clinical applications of saffron (Crocus sativus) and its constituents: a review. Drug Res. 2015;65(6):287-95. 
32. Pitsikas N. The effect of Crocus sativus L. and its constituents on memory: Basic studies and clinical applications. Evidence-based Complementary and Alternative Medicine. 2015:article number 926284, 2015.

33. Moher D, Liberati A, Tetzlaff J, Altman DG, Group P. Preferred reporting items for systematic reviews and meta-analyses: the PRISMA statement. PLoS Med. 2009;6(7):e1000097.

34. Higgins JP, Altman DG. Assessing risk of bias in included studies. In: Cochrane Handbook for Systematic Reviews of Interventions: Cochrane Book Series. Volume 1. 1st edition. Edited by Higgins JP. and Green S. New Jersey: Wiley; 2008. p. 187-241.

35. Rockwood K, Fay S, Gorman M, Carver D, Graham JE. The clinical meaningfulness of ADAS-cog changes in Alzheimer's disease patients treated with donepezil in an open-label trial. BMC Neurol. 2007;7(1):26.

36. Schmidt M, Betti G, Hensel A. Saffron in phytotherapy: pharmacology and clinical uses. Wien Med Wochenschr. 2007:157(13-14):315.

37. Broadhead G, Chang A, Grigg J, McCluskey P. Efficacy and safety of saffron supplementation: current clinical findings. Crit Rev Food Sci Nutr. 2016; 56(16):2767-76.

38. Bhargava V. Medicinal uses and pharmacological properties of Crocus sativus Linn. (Saffron). Int J Pharm Pharm Sci. 2011;3:22-6.

39. Adalier $\mathrm{N}$, Parker $\mathrm{H}$. Vitamin $\mathrm{E}$, turmeric and saffron in treatment of Alzheimer's disease. Antioxidants. 2016:5(4):40.

40. Khazdair MR, Boskabady MH, Hosseini M, Rezaee R, Tsatsakis AM. The effects of Crocus sativus (saffron) and its constituents on nervous system: a review. Avicenna J Phytomedicine. 2015;5(5):376-91.

\section{Publisher's Note}

Springer Nature remains neutral with regard to jurisdictional claims in published maps and institutional affiliations.

Ready to submit your research? Choose BMC and benefit from:

- fast, convenient online submission

- thorough peer review by experienced researchers in your field

- rapid publication on acceptance

- support for research data, including large and complex data types

- gold Open Access which fosters wider collaboration and increased citations

- maximum visibility for your research: over $100 \mathrm{M}$ website views per year

At BMC, research is always in progress.

Learn more biomedcentral.com/submissions 